Pacific Journal of Mathematics

GEOMETRIC OVERCONVERGENCE OF RATIONAL 


\title{
GEOMETRIC OVERCONVERGENCE OF RATIONAL FUNCTIONS IN UNBOUNDED DOMAINS
}

\author{
E. B. SAFF AND R. S. VARGA
}

Dedicated to the memory of our teacher, Professor Joseph L. Walsh

The basic aim of this paper is to study the phenomenon of overconvergence for rational functions converging geometrically on $[0,+\infty)$.

1. Introduction. The classical results of Bernstein, Walsh, Gončar, and others concerning the overconvergence of rational functions are roughly of the following type (cf. [18]): It is assumed that

(i) $f(z)$ is defined (finite) on some compact set $E$ in the complex plane $\mathbf{C}$;

(ii) $\left\{r_{n}(z)\right\}_{n=1}^{\infty}$ is a sequence of rational functions of respective degrees $n$ which converge geometrically to $f$ on $E$, i.e.,

$$
\varlimsup_{n \rightarrow \infty}\left\{\left\|f-r_{n}\right\|_{L_{\infty}(E)}\right\}^{1 / n}<1
$$

and

(iii) the set of poles of the sequence $\left\{r_{n}(z)\right\}_{n=1}^{\infty}$ has no accumulation points on $E$.

It is then concluded that

(iv) the sequence $\left\{r_{n}(z)\right\}_{n=1}^{\infty}$ converges geometrically to an analytic extension of $f$ on some open set in the plane containing $E$.

The aim of the present paper is to investigate the phenomenon of overconvergence in the case where $E$ is a closed line segment $[a, b]$ and the hypothesis (iii) above is weakened to allow accumulation points of poles at the endpoints of $E$, i.e., assumption (iii) is replaced by

(iii)' the set of poles of the sequence $\left\{r_{n}(z)\right\}_{n=1}^{\infty}$ has no accumulation points on the open subinterval $(a, b)$ of $E=[a, b]$.

Of course with the hypotheses (i), (ii), and (iii)', we must modify conclusion (iv) to read

(iv) ${ }^{\prime}$ the sequence $\left\{r_{n}(z)\right\}_{n=1}^{\infty}$ converges geometrically to an analytic extension of $f$ on some open set in the plane containing $(a, b)$. 
For the precise statements of such results on "angular overconvergence" it is sufficient to take $E=[0,+\infty)$, because any interval $[a, b]$ can be mapped onto $[0,+\infty)$ by means of a bilinear transformation, and such bilinear transformations preserve rational functions of degree $n$. For example, one of the results which we prove asserts that if rational functions $r_{n}(z)$ of respective degrees $n$ converge geometrically on $E=[0,+\infty)$, and the poles of the $r_{n}(z)$ lie outside an infinite sector of the form

$$
\left\{z \in \mathbf{C}:|\arg z|<\phi_{1}\right\}, \quad 0<\phi_{1} \leqq \pi,
$$

then the $r_{n}(z)$ converge geometrically on some smaller infinite sector

$$
\left\{z \in \mathbf{C}:|\arg z|<\phi_{2}\right\}, \quad 0<\phi_{2}<\phi_{1} \text {. }
$$

It is important to note that a number of results have appeared in the literature ([8], [10], [11]) which give classes of functions $f$ and examples of approximating rational functions $r_{n}(z)$ for which condition (ii) above is satisfied on $E=[0,+\infty)$. Furthermore, for some special sequences of approximating rational functions, the existence of pole-free open sets (in the plane) containing $(0,+\infty)$ follows from the results in [17], [12], [13], among others. Hence the main results of this paper, which we state in $\$ 2$, have immediate applications. These applications will be discussed primarily in $\$ 3$.

2. Statements of main results. We now introduce the necessary notation and state our main results. Their proofs will be given in $\$ 4$.

For an arbitrary set $A$ in the complex plane $\mathbf{C}$ we denote by $\|\cdot\|_{A}$ the sup norm on $A$, i.e.,

$$
\|f\|_{A}:=\sup \{|f(z)|: z \in A\} .
$$

We use the symbol $\pi_{n}$ to denote the set of all complex polynomials in the variable $z$ having degree at most $n$, and let $\pi_{n, n}$ denote the set of all complex rational functions $r_{n}(z)$ of the form

$$
r_{n}(z)=\frac{p_{n}(z)}{q_{n}(z)}, \quad \text { where } \quad p_{n} \in \pi_{n}, \quad q_{n} \in \pi_{n}, \quad q_{n} \not \equiv 0
$$

The first three results which we state concern pole-free regions whose boundaries are parallel to the ray $E=[0,+\infty)$ at $x=+\infty$. It is convenient in this regard to introduce the set $\mathscr{H}$ which consists of all real nonnegative continuous functions $h$ on $[0,+\infty)$ such that for $x$ large, $h(x)>0$, and $h^{\prime}(x)$ exists, is nonnegative, and satisfies 


$$
\lim _{x \rightarrow+\infty} h^{\prime}(x)=0
$$

Corresponding to each $h \in \mathscr{H}$ we define generically the set $E_{s}(h)$, $0 \leqq s \leqq 1$, in the complex plane by

$$
E_{s}(h):=\{z=x+i y: x \geqq 0 \text { and }|y| \leqq s h(x)\} \text {. }
$$

Notice that, by condition (2.1), the boundary of each set $E_{s}(h)$ defined in (2.2) makes an angle of zero with the positive real axis at $x=+\infty$.

Our first result is the following:

THEOREM 2.1. Assume that for a function $f$, defined and finite on $[0,+\infty)$, there exists a sequence of rational functions $\left\{r_{n}\right\}_{n=1}^{\infty}$, with $r_{n} \in \pi_{n, n}$ for all $n \geqq 1$, and a real number $q>1$ such that

$$
\varlimsup_{n \rightarrow \infty}\left\{\left\|f-r_{n}\right\|_{\{0,+\infty)}\right\}^{1 / n}<\frac{1}{q}<1 \text {. }
$$

Assume further that for some function $h \in \mathscr{H}$ the interior of the region $E_{1}(h)$ (defined in (2.2)) contains no poles of the $r_{n}(z)$ for all $n$ sufficiently large. Then for every $d$ satisfying the inequality

$$
0<d<\frac{\sqrt{q}-1}{\sqrt{q}+1}<1
$$

there exists a bounded subset $K_{d}$ of $E_{d}(h)$ and an analytic function $F(z)$ on $E_{d}(h)-K_{d}$ with $F(x)=f(x)$ for all real $x$ in this set, such that $\left\{r_{n}(z)\right\}_{n=1}^{\infty}$ converges geometrically to $F(z)$ on $E_{d}(h)-K_{d}$. Moreover

$$
\varlimsup_{n \rightarrow \infty}\left\{\left\|F-r_{n}\right\|_{E_{d}(h)-K_{d}}\right\}^{1 / n}<\frac{1}{q} \cdot\left(\frac{1+d}{1-d}\right)^{2}<1 .
$$

The next result shows that in certain cases the conclusion of Theorem 2.1 can hold on the whole set $E_{d}(h)$, rather than on $E_{d}(h)-K_{d}$.

COROllary 2.2. Assume that for a continuous function $g(\not \equiv 0)$ on $[0,+\infty)$ there exists a sequence of polynomials $\left\{p_{n}\right\}_{n=1}^{\infty}$, with $p_{n} \in \pi_{n}$ for all $n \geqq 1$, and a real number $q>1$ such that

$$
\varlimsup_{n \rightarrow \infty}\left\{\left\|\frac{1}{g}-\frac{1}{p_{n}}\right\|_{(0,+\infty)}\right\}^{1 / n} \leqq \frac{1}{q}<1 .
$$


Then, as is known [7, Theorem 3], there exists an entire function $G(z)$ of finite order with $G(x)=g(x)$ for all $x \geqq 0$. Next assume that for some function $h \in \mathscr{H}$, with $h(x)>0$ for all $x>0$, the interior of the region $E_{1}(h)$ (defined in (2.2)) contains no zeros of $p_{n}(z)$ for all $n$ large. If $d$ satisfies (2.4) and if $G$ is nonzero on the vertical segment $\{z=i y:|y| \leqq d h(0)\}$, then

$$
\varlimsup_{n \rightarrow \infty}\left\{\left\|\frac{1}{G}-\frac{1}{p_{n}}\right\|_{E_{d}(h)}\right\}^{1 / n} \leqq \frac{1}{q}\left(\frac{1+d}{1-d}\right)^{2}<1
$$

As a concrete application of Corollary 2.2, we first recall from Meinardus and Varga [8] that

$$
\lim _{n \rightarrow \infty}\left\{\left\|e^{-x}-\frac{1}{s_{n}(x)}\right\|_{[0,+\infty)}\right\}^{1 / n}=\frac{1}{2}
$$

where $s_{n}(z)=\sum_{k=0}^{n} z^{k} / k$ ! denotes the familiar $n$th partial sum of $e^{z}$. It is further known from Saff and Varga [12] that for

$$
\hat{h}(x)=2(x+1)^{1 / 2}
$$

the region

$$
E_{1}(\hat{h})=\left\{z=x+i y: x \geqq 0,|y| \leqq 2(x+1)^{1 / 2}\right\}
$$

contains no zeros of the $s_{n}(z)$ for all $n$. Note that $\hat{h} \in \mathscr{H}$, and that with $G(z)=e^{z}$ (so that $G$ is nonzero at every finite point $z$ ), with $p_{n} \equiv s_{n}$ for all $n \geqq 1$, and with $q=2$, the hypotheses of Corollary 2.2 are all fulfilled. Thus for any $d$ satisfying $0<d<(\sqrt{2}-1) /(\sqrt{2}+1)$, we have from (2.7) that

$$
\varlimsup_{n \rightarrow \infty}\left\{\left\|e^{-z}-\frac{1}{S_{n}(z)}\right\|_{E_{d}(\hat{h})}\right\}^{1 / n} \leqq \frac{1}{2}\left(\frac{1+d}{1-d}\right)^{2}<1,
$$

which is effectively the result of [11, Theorem 4.1]. We remark that for any $d>0$ the set

$$
E_{d}(\hat{h})=\left\{z=x+i y: x \geqq 0,|y| \leqq 2 d(x+1)^{1 / 2}\right\}
$$

is an unbounded parabolic region truncated at the origin.

As a consequence of Corollary 2.2 and of the results in [12], similar overconvergence results in unbounded parabolic regions also hold for each column of the Padé table for $e^{-z}$, i.e., for the Padé approximants $\left\{R_{\nu, n}(z)\right\}_{n=1}^{\infty}$ where the degree, $\nu$, of the numerator is fixed. 
Applications of Corollary 2.2 can in fact be made to a certain class of entire functions which contains the above example, and this will be described in the next section.

From Corollary 2.2 it is possible to deduce the following result which concerns geometric convergence on related unbounded sets whose widths grow more slowly at infinity.

COROLlary 2.3. With the hypotheses of Corollary 2.2, assume that $c(x)$ is a nonnegative continuous function on $[0,+\infty)$ with $c(x)<h(x)$ for all $x>0$, such that

$$
\lim _{x \rightarrow+\infty} \frac{c(x)}{h(x)}=0
$$

and let

$$
\mathscr{C}:=\{z=x+i y: x \geqq 0,|y| \leqq c(x)\} .
$$

If $G$ is nonzero on the segment $\{z=i y:|y| \leqq c(0)\}$, then

$$
\varlimsup_{n \rightarrow \infty}\left\{\left\|\frac{1}{G}-\frac{1}{p_{n}}\right\|_{\mathscr{C}}\right\}^{1 / n}=\varlimsup_{n \rightarrow \infty}\left\{\left\|\frac{1}{g}-\frac{1}{p_{n}}\right\|_{[0,+\infty)}\right\}^{1 / n} .
$$

The remaining results concern overconvergence on regions having a positive angle at infinity. In stating them it is convenient to introduce the sets $S(\theta, \mu)$ and $S(\theta)$ defined by

$$
\begin{aligned}
S(\theta, \mu): & =\{z:|\arg z|<\theta,|z|>\mu\}, \\
S(\theta): & =\{z:|\arg z|<\theta\} .
\end{aligned}
$$

THEOREM 2.4. Assume that for a function $f$, defined and finite on $[0,+\infty)$, there exists a sequence of rational functions $\left\{r_{n}\right\}_{n=1}^{\infty}$, with $r_{n} \in \pi_{n, n}$ for all $n \geqq 1$, and a real number $q>1$ such that

$$
\varlimsup_{n \rightarrow \infty}\left\{\left\|f-r_{n}\right\|_{[0,+\infty)}\right\}^{1 / n}<\frac{1}{q}<1 .
$$

Assume further that for some $\theta_{0}$ and $\mu_{0}$, with $0<\theta_{0} \leqq \pi, \mu_{0}>0$, the region $S\left(\theta_{0}, \mu_{0}\right)$ (defined in (2.16)) contains no poles of the $r_{n}(z)$ for all $n$ large. Then for every $\theta$ satisfying the inequality

$$
0<\theta<4 \tan ^{-1}\left\{\left(\frac{\sqrt{q}-1}{\sqrt{q}+1}\right) \cdot \tan \left(\frac{\theta_{0}}{4}\right)\right\},
$$


there exists $a \mu=\mu(\theta)>0$ and an analytic function $F(z)$ on the closure $\bar{S}(\theta, \mu)$ with $F(x)=f(x)$ for all real $x$ in this set, such that $\left\{r_{n}(z)\right\}_{n=1}^{\infty}$ converges geometrically to $F(z)$ on $\bar{S}(\theta, \mu)$. Moreover

$$
\varlimsup_{n \rightarrow \infty}\left\{\left\|F-r_{n}\right\|_{\bar{S}(\theta, \mu)}\right\}^{1 / n}<\frac{1}{q} \cdot\left\{\frac{\sin \left[\frac{1}{4}\left(\theta_{0}+\theta\right)\right]}{\sin \left[\frac{1}{4}\left(\theta_{0}-\theta\right)\right]}\right\}^{2}<1 .
$$

It is interesting to note that while Theorem 2.1 cannot be deduced from Theorem 2.4, the former result can be considered as a limiting case of the latter. Indeed, for the situation of Theorem 2.1, we regard $\theta_{0}$ and $\theta$ as functions of $x$ which tend to zero as $x \rightarrow+\infty$; specifically, we define $\theta_{0}$ and $\theta$ by the equations

$$
\tan \theta_{0}=\frac{h(x)}{x}, \quad \tan \theta=\frac{d h(x)}{x} .
$$

Then, on writing (2.19) in the equivalent form

$$
\frac{\tan (\theta / 4)}{\tan \left(\theta_{0} / 4\right)}<\frac{\sqrt{q}-1}{\sqrt{q}+1}
$$

and taking the limit as $x \rightarrow+\infty$, we derive the condition

$$
\lim _{x \rightarrow+\infty} \frac{\tan (\theta / 4)}{\tan \left(\theta_{0} / 4\right)}=\lim _{x \rightarrow+\infty} \frac{\tan \theta}{\tan \theta_{0}}=d<\frac{\sqrt{q}-1}{\sqrt{q}+1}
$$

which is the same as inequality (2.4) of Theorem 2.1.

Using Theorem 2.4 we can deduce the following analogs of Corollaries 2.2 and 2.3:

Corollary 2.5. Let the functions $g, G$, and the sequence of polynomials $\left\{p_{n}\right\}_{n=1}^{\infty}$ be as in Corollary 2.2 (so that, in particular, inequality (2.6) holds). Assume further that no zeros of $p_{n}$ lie in the infinite sector $S\left(\theta_{0}\right)$ (defined in (2.17)), $0<\theta_{0} \leqq \pi$, for all $n$ sufficiently large, and that $g(0) \neq 0$. If $\theta$ satisfies (2.19), then on the closure $\bar{S}(\theta)$,

$$
\varlimsup_{n \rightarrow \infty}\left\{\left\|\frac{1}{G}-\frac{1}{p_{n}}\right\|_{\bar{s}(\theta)}\right\}^{1 / n} \leqq \frac{1}{q}\left\{\frac{\sin \left[\frac{1}{4}\left(\theta_{0}+\theta\right)\right]}{\sin \left[\frac{1}{4}\left(\theta_{0}-\theta\right)\right]}\right\}^{2}<1 .
$$

Corollary 2.6. Let the functions $g, G$, and the sequence of polynomials $\left\{p_{n}\right\}_{n=1}^{\infty}$ be as in Corollary 2.2 (so that, in particular, inequality (2.6) holds). Assume that no zeros of $p_{n}$ lie in $S\left(\theta_{0}\right), 0<\theta_{0} \leqq \pi$, for all $n$ sufficiently large, and that $g(0) \neq 0$. Then for any nonnegative continuous 
function $c(x)$ on $[0,+\infty)$ such that $c(x)=o(x)$ as $x \rightarrow+\infty$ and such that (i) $c(0)=0$ if $\theta_{0}=\pi / 2$, (ii) $c(x)<x \tan \left(\theta_{0}\right)$ for $x>0$ if $0<\theta_{0}<\pi / 2$, we have

$$
\varlimsup_{n \rightarrow \infty}\left\{\left\|\frac{1}{G}-\frac{1}{p_{n}}\right\|_{\zeta}\right\}^{1 / n}=\varlimsup_{n \rightarrow \infty}\left\{\left\|\frac{1}{g}-\frac{1}{p_{n}}\right\|_{[0,+\infty)}\right\}^{1 / n},
$$

where the region $\mathscr{b}$ is defined as in (2.14).

If, in Corollary 2.5, we weaken the hypothesis by replacing the reciprocals of polynomials, $1 / p_{n}$, by arbitrary rational functions $r_{n} \in \pi_{n, n}$ whose poles omit a full sector, then we obtain the following less specific conclusion:

THEOREM 2.7. Assume that for a function $f$, defined and finite on $[0,+\infty)$, there exists a sequence of rational functions $\left\{r_{n}\right\}_{n=1}^{\infty}$, with $r_{n} \in \pi_{n, n}$ for all $n \geqq 1$, and a real number $q>1$ such that inequality (2.18) holds. Suppose further that the infinite sector $S\left(\theta_{0}\right)$ (defined in (2.17)), $0<\theta_{0} \leqq \pi$, contains no poles of the $r_{n}(z)$ for all $n$ large. Then there exists $a \theta, 0<\theta<\theta_{0}$, and a function $F(z)$ analytic on the sector $S(\theta)$, continuous on $\bar{S}(\theta)$, with $F(x)=f(x)$ for all $x \geqq 0$, such that $\left\{r_{n}(z)\right\}_{n=1}^{\infty}$ converges geometrically to $F(z)$ on $\bar{S}(\theta)$.

Theorem 2.7 has an important application to the problem (raised at the International Conference on Approximation Theory, Maryland, 1970) of finding a sequence of rational functions which converges geometrically to $e^{-z}$ in an infinite sector. It is well-known that the sequence $1 / s_{n}(z), s_{n}(z)=\sum_{0}^{n} z^{k} / k$ !, does not have this property because no infinite sector is devoid of zeros of $s_{n}(z)$ for all $n$ large (cf. [3] or [14]). However, it is shown by the authors in [11] and [13], that certain sequences of Padé approximants of $e^{-z}$ converge geometrically on $[0,+\infty)$ to $e^{-x}$, and furthermore have all their poles outside some infinite sector $\left\{z:|\arg z|<\theta_{0}\right\}$. Hence, by Theorem 2.7 , such a sequence must converge geometrically to $e^{-z}$ on some infinite sector $\{z:|\arg z|<\theta\}$, $0<\theta<\theta_{0}$. The precise details of this application shall be reserved for a later occasion.

The last result of this section concerns rational functions which converge faster than geometrically on $[0,+\infty)$, i.e.,

$$
\lim _{n \rightarrow \infty}\left\{\left\|f-r_{n}\right\|_{[0,+\infty)}\right\}^{1 / n}=0 .
$$

COROllary 2.8. If in Theorem 2.7, the assumption of inequality (2.18) is replaced by (2.23), then the sequence $\left\{r_{n}(z)\right\}_{n=1}^{\infty}$ converges faster than geometrically on every closed sector $\bar{S}(\theta), 0<\theta<\theta_{0}$, i.e., 


$$
\lim _{n \rightarrow \infty}\left\{\left\|F-r_{n}\right\|_{\bar{s}(\theta)}\right\}^{1 / n}=0 .
$$

3. Some applications. In order to apply results such as Corollaries 2.2 and 2.3 we first need conditions on the entire function $G(z)$ which insure that there exists a sequence of polynomials $p_{n}$, with $p_{n} \in \pi_{n}$ for all $n \geqq 1$, such that

$$
\varlimsup_{n \rightarrow \infty}\left\{\left\|\frac{1}{G}-\frac{1}{p_{n}}\right\|_{[0,+\infty)}\right\}^{1 / n}<1 .
$$

Second, we need a specific result, like that of (2.9), which asserts that for an appropriate function $h \in \mathscr{H}$, the interior of the region $E_{1}(h)$ defined in (2.2) is free of zeros of the polynomials $p_{n}$ in (3.1) for all $n$ large. Results of both these types are already known for the case where the $p_{n}$ are the $n$th partial sums of the Maclaurin expansion for $G$. In order to state these results we remind the reader of some standard terminology.

If $g(z)=\sum_{k=0}^{\infty} a_{k} z^{k}$ is an entire function, we let $M_{g}(r):=$ $\max \{|g(z)|:|z|=r\}$ denote its maximum modulus function, and let $\rho=\rho_{g}$ denote the order of $g$ (for nonconstant $g$ ), i.e., (cf. [2, p. 8], [15, p. 34])

$$
\rho=\varlimsup_{r \rightarrow+\infty} \frac{\ln \left(\ln M_{g}(r)\right)}{\ln r} .
$$

Furthermore, an entire function $g(z)$ of order $\rho, 0<\rho<\infty$, is said to be of perfectly regular growth (cf. [15, p. 44]) if there exists a real $B>0$ such that

$$
0<B=\lim _{r \rightarrow+\infty} \frac{\ln M_{g}(r)}{r^{\rho}}
$$

We remark that if a nonconstant entire function $g$ satisfies a linear differential equation with rational function coefficients, then $g$ is necessarily of perfectly regular growth (cf. [15, p. 108]).

We now state a result which gives sufficient conditions for geometric convergence on $[0,+\infty)$.

THEOREM 3.1 (Meinardus and Varga [8]). Let $g(z)=\sum_{k=0}^{\infty} a_{k} z^{k}$ be an entire function of perfectly regular growth $(\rho, B)$ with real nonnegative coefficients $a_{k}$. Then

$$
\lim _{n \rightarrow \infty}\left\{\left\|\frac{1}{g}-\frac{1}{S_{n}}\right\|_{[0,+\infty)}\right\}^{1 / n}=\frac{1}{2^{1 / \rho}}<1
$$


where $s_{n}(z)=\sum_{k=0}^{n} a_{k} z^{k}$ denotes the $n$th partial sum of the Maclaurin expansion for $g$.

Concerning zero-free regions for the partial sums $s_{n}$ we state a previously unpublished result from one of the author's thesis [17]. For related published results see [16].

THEOREM 3.2. Let $\mathscr{S}$ denote the set of all entire functions $g(z)=$ $\sum_{k=0}^{\infty} a_{k} z^{k}$ for which

(i) $\quad a_{0}>0$ and $a_{k} \geqq 0$ for all $k \geqq 1$;

(ii) if $a_{m}=0$, then $a_{m+2 \jmath}=0$ for every $j \geqq 1$;

(iii) if $K:=\left\{k: a_{k}>0\right.$ and $\left.a_{k+2}>0\right\}$ is nonempty, then

$$
\inf _{k \in K}\left\{\frac{a_{k}}{(k+1)(k+2) a_{k+2}}\right\}>0 \text {. }
$$

Then, for $g \in \mathscr{S}$, there exists a nondecreasing continuous function $h_{g}$ defined on $[0,+\infty)$ with $h_{g}(0)>0$, such that $g(z)$ and all its partial sums $s_{n}(z)=\sum_{k=0}^{n} a_{k} z^{k}, n \geqq 1$, have no zeros in

$$
\left\{z=x+i y: x \geqq 0 \text { and }|y| \leqq h_{g}(x)\right\} .
$$

Moreover, for each $g \in \mathscr{S}$, the order $\rho_{g}$ of $g$ satisfies $0 \leqq \rho_{g} \leqq 1$.

We remark that the set $\mathscr{S}$ of Theorem 3.2 contains many familiar elements. For example, $u(z)=e^{z}, v(z)=\cosh (\sqrt{z})=\Sigma_{0}^{\infty} z^{k} /(2 k)$ !, the modified Bessel functions $J_{n}(i z) /(i z)^{n}$ for any $n \geqq 0$, and the hypergeometric function ${ }_{1} F_{1}(c ; d ; z)$ with $c>0, d>0$, are easily seen to be elements of $\mathscr{S}$.

If $W_{g}$ denotes the nonempty (from Theorem 3.2) collection of all positive nondecreasing continuous functions $h_{g}$ on $[0,+\infty)$ for which $g(z)$ and all its partial sums $s_{n}(z), n \geqq 1$, have no zeros in the region defined by (3.6), then we define the (maximal) width function $H_{g}(x)$ by

$$
H_{g}(x):=\sup \left\{h_{g}(x): h_{g} \in \mathscr{W}_{g}\right\}, \text { for each } x \geqq 0 .
$$

The function $H_{g}(x)$ so defined is clearly nondecreasing on $[0,+\infty)$, and $g(z)$ and all its partial sums $s_{n}(z)$ have no zeros in the interior of the region defined by

$$
\left\{z=x+i y: x \geqq 0 \text { and }|y| \leqq H_{g}(x)\right\} .
$$

Moreover, if $g$ is of order $\rho_{g}>0$, then a result of Carlson [3] states that no proper sector, with vertex at the origin, can be devoid of zeros of the 
partial sums $s_{n}$, for all $n$ large. Consequently, when $\rho_{g}>0, H_{g}(x)$ is finite for all finite $x \geqq 0$.

The next corollary provides lower bounds for $H_{g}(x)$ for particular elements in $\mathscr{S}$.

COROLlaRY 3.3. Let $g(z)=\sum_{0}^{\infty} a_{k} z^{k}$ be an entire function such that $a_{k}>0$ for all $k$ and such that

$$
\inf _{k \geqq 1}\left\{\frac{a_{k}}{k^{2} a_{k+1}}\right\}>0 \text {. }
$$

Then $g \in \mathscr{S}$ and its associated width function $H_{g}$ of (3.7) satisfies, for some constant $c>0$,

$$
H_{g}(x) \geqq c x^{1 / 2}, \text { for all } x \geqq 0 \text {. }
$$

Proof. It is trivial to verify that $g(z)=\sum_{0}^{\infty} a_{k} z^{k} \in \mathscr{S}$. Furthermore, it follows from the hypotheses above that the entire function $f$ defined by $f(z):=\sum_{0}^{\infty} a_{k} z^{2 k}$ is also in $\mathscr{S}$. Thus, from Theorem 3.2, we can associate with $f$ a continuous nondecreasing function $h_{f}$ defined on $[0,+\infty)$, with $h_{f}(0)>0$, such that $f$ and all its partial sums $S_{n}(z)$ have no zeros in

$$
\mathscr{F}:=\left\{z=x+i y: x \geqq 0 \text { and }|y| \leqq h_{f}(x)\right\} \text {. }
$$

But if $s_{n}(z)$ denotes the $n$th partial sum of $g(z)$, then $s_{n}\left(z^{2}\right)=S_{2 n}(z)$ for all $n=1,2, \cdots$, which allows us to relate the corresponding zeros of the partial sums of $g$ with those of $f$. Thus, defining

$$
\mathscr{G}:=\left\{z^{2}: z \in \mathscr{F}\right\}
$$

then $g$ and all its partial sums $s_{n}$ have no zeros in $\mathscr{G}$. Now, since $h_{f}(0)>0$ and $h_{f}$ is nondecreasing on $[0,+\infty)$, then evidently

$$
\mathscr{G} \supset\left\{z^{2}: z=x+i y, x \geqq 0 \text { and }|y| \leqq h_{f}(0)\right\} .
$$

Thus, if $H_{g}$ is the associated width function for $g$, the above inclusion implies that

$$
H_{g}(t) \geqq 2 h_{f}(0)\left(t+h_{f}^{2}(0)\right)^{1 / 2} \geqq 2 h_{f}(0) t^{1 / 2}, \quad \text { for all } t \geqq 0,
$$

which is the desired result of (3.10).

As previously noted, $u(z)=e^{z}$, of order $\rho_{u}=1$, and $v(z)=$ $\cosh (\sqrt{z})$, of order $\rho_{v}=1 / 2$, are elements of the set $\mathscr{S}$, and furthermore 
each is of perfectly regular growth. Moreover, for $u(z)=e^{z}$, the authors' result of (2.9) implies that

$$
H_{u}(x) \geqq 2(x+1)^{1 / 2}, \text { for all } x \geqq 0 .
$$

Also, applying Corollary 3.3 to $v(z)=\cosh (\sqrt{z})$ gives

$$
H_{v}(x) \geqq c x^{1 / 2}, \text { for all } x \geqq 0 \text {. }
$$

However, we believe that this last inequality can be improved. In fact, we conjecture more generally that, for any element $g \in \mathscr{S}$ of perfectly regular growth, its associated width function satisfies

$$
H_{g}(x) \geqq c x^{\left(2-\rho_{g}\right) / 2}, \text { for all } x \geqq 0 \text {. }
$$

As a consequence of Theorems 3.1 and 3.2, which apply to both $e^{z}$ and $\cosh (\sqrt{z})$, we have the following application of Corollary 2.2.

COROllary 3.4. For any $g \in \mathscr{S}$ of order $\rho>0$ which is of perfectly regular growth, let $H_{g}$ be its associated nondecreasing width function of (3.7), and let $h \in \mathscr{H}$ be any positive function for which $h(x) \leqq H_{g}(x)$ for all $x \geqq 0$. Then for

$$
0<d<\left(2^{1 / 2 \rho}-1\right) /\left(2^{1 / 2 \rho}+1\right)
$$

we have

$$
\varlimsup_{n \rightarrow \infty}\left\{\left\|\frac{1}{g}-\frac{1}{s_{n}}\right\|_{E_{d}(h)}\right\}^{1 / n} \leqq \frac{1}{2^{1 / \rho}}\left(\frac{1+d}{1-d}\right)^{2}<1,
$$

where the region $E_{d}(h)$ is defined as in (2.2), and $s_{n}(z)$ denotes the $n$th partial sum of $g(z)$.

Proof. Because $g \in \mathscr{S}$ implies that the Maclaurin coefficients of $g$ are all nonnegative, and because $g$ is assumed to be of perfectly regular growth, then the conclusion (3.4) of Theorem 3.1 is valid. Next, by the definition of $H_{g}(x)$ and the fact that $h(x) \leqq H_{g}(x)$ for all $x \geqq 0$, it follows that $g$ and all its partial sums $s_{n}$ have no zeros in the interior of the region $E_{1}(h)$. Consequently, applying Corollary 2.2 , with $q=2^{1 / \rho}$, gives the desired result of (3.11).

We remark that the existence of a function $h \in \mathscr{H}$ satisfying the conditions of Corollary 3.4 is obvious. As a simple example, take $h_{g}$ of Theorem 3.2 and set $h(x) \equiv h_{g}(0)$. 
Concerning rational approximation to entire functions of order $\rho=0$, it has been shown in [7, Thm. 7] and in [4, Thm. 2] that if $g$ is an entire function of order zero and satisfies certain growth and coefficient restrictions, then

$$
\lim _{n \rightarrow \infty}\left\{\inf _{p \in \pi_{n}}\left\|\frac{1}{g}-\frac{1}{p} \cdot\right\|_{[0,+\infty)}\right\}^{1 / n}=0
$$

As an illustration of how our techniques apply to such situations, we present

Proposition 3.5. Let $g(z)=\sum_{k=0}^{\infty} z^{k} / a^{k^{2}}$, where $a \geqq 2$, and let $s_{n}(z)=\sum_{k=0}^{n} z^{k} / a^{k^{2}}$. Then, on every closed sector $\bar{S}(\theta)$ (defined in (2.17)) with $0<\theta<\pi$, we have

$$
\lim _{n \rightarrow \infty}\left\{\left\|\frac{1}{g}-\frac{1}{s_{n}}\right\|_{\bar{s}(\theta)}\right\}^{1 / n^{2}}=\frac{1}{\sqrt{a}}
$$

Of course, for the functions of Proposition 3.5, we see that the conclusion of (3.13) is far stronger, and implies the result of (3.12), as a special case.

As the proof of Proposition 3.5 follows from the methods of proof of the main results in $\S 2$, we shall defer it to the next section.

4. Proofs of new results. We now present the proofs of the main results given in $\$ 2$. It is convenient in this regard to first state a particular case of Walsh's Lemma [18, p. 250].

LEMmA 4.1. Let $r_{n}(z) \in \pi_{n, n}$ have poles in the extended complex plane at the points $\beta_{1}, \beta_{2}, \cdots, \beta_{m}, m \leqq n$, (listed according to multiplicity). If

$$
\left\|r_{n}(z)\right\|_{[-1,+1]} \leqq M<\infty
$$

then for $z \notin[-1,+1]$,

$$
\left|r_{n}(z)\right| \leqq M \prod_{k=1}^{m}\left|\frac{1-\overline{\Phi\left(\beta_{k}\right)} \cdot \Phi(z)}{\Phi(z)-\Phi\left(\beta_{k}\right)}\right|
$$

where $w=\Phi(z)$ is the inverse of the function

$$
z=\Psi(w)=\frac{1}{2}\left(w+\frac{1}{w}\right)
$$


which maps $|w|>1$ in the $w$-plane one-to-one onto the exterior of $[-1,+1]$ in the $z$-plane, so that $\Psi(\infty)=\infty$. Moreover, if $C_{R}$ denotes generically the elliptical level curve $|\Phi(z)|=R>1$ in the $z$-plane, and if all the points $\beta$, lie on or exterior to $C_{A}$, then for any $1 \leqq S \leqq A$, .

$$
\left\|r_{n}(z)\right\|_{\bar{C}_{s}} \leqq M\left(\frac{A S-1}{A-S}\right)^{n}
$$

where $\bar{C}_{S}$ denotes the closed interior of $C_{s}$ in the $z$-plane.

We remark that if $r_{n}=p_{n} \in \pi_{n}$, then (4.2) is valid for any $A>S$, and letting $A \rightarrow \infty$ in (4.2) gives the familiar result of Bernstein (cf. [6, p. 92]),

$$
\left\|p_{n}(z)\right\|_{\bar{C}_{s}} \leqq M S^{n}, \quad \text { if } \quad\left\|p_{n}(z)\right\|_{[-1,+1]} \leqq M
$$

which can also be deduced from (4.1) with all $\beta_{\imath}=\infty$.

Proof of Theorem 2.1. For $\delta>0$, set $q_{1}:=q+\delta, q_{2}:=q+2 \delta$, and select $\delta>0$ sufficiently small so that (cf. (2.3))

$$
\varlimsup_{n \rightarrow \infty}\left\{\left\|f-r_{n}\right\|_{[0,+\infty)}\right\}^{1 / n}<\frac{1}{q_{2}}<\frac{1}{q_{1}}<\frac{1}{q} .
$$

Next, for any $\beta \geqq 1$, define

$$
A^{*}(\beta):=\frac{1}{\beta}+\sqrt{\frac{1}{\beta^{2}}+1}, \text { and } \rho^{*}(\beta):=A^{*}(\beta / d),
$$

where $d$ is fixed and satisfies (2.4). An easy calculation with these definitions then shows that

$$
\lim _{\beta \rightarrow \infty}\left(\frac{A^{*}(\beta) \rho^{*}(\beta)-1}{A^{*}(\beta)-\rho^{*}(\beta)}\right)=\frac{1+d}{1-d} .
$$

Thus, we can choose a finite $\hat{\beta} \geqq 1$, dependent on $\delta$, such that

$$
1<\left(\frac{A^{*}(\hat{\beta}) \rho^{*}(\hat{\beta})-1}{A^{*}(\hat{\beta})-\rho^{*}(\hat{\beta})}\right)^{2}<\frac{q_{2}}{q_{1}}\left(\frac{1+d}{1-d}\right)^{2} .
$$

From (4.3) there exists an $n_{0}$, depending on $\delta$, such that

$$
\left\|f-r_{n}\right\|_{[0,+\infty)}<\frac{1}{q_{2}^{n}}, \text { for all } n \geqq n_{0} .
$$


Thus, by the triangle inequality, it follows that

$$
\left\|r_{n}-r_{n-1}\right\|_{(0,+\infty)}<\frac{\left(q_{2}+1\right)}{q_{2}^{n}}, \text { for all } n>n_{0} .
$$

Since by hypothesis $h \in \mathscr{H}$, there exists a $t_{1} \geqq 0$ such that $h(t)>0$ for all $t \geqq t_{1}$. Consider then the line segment of the real axis

$$
m(t):=[t-\hat{\beta} h(t), t+\hat{\beta} h(t)]
$$

for any $t \geqq t_{1}$. With L'Hospital's rule, the condition of (2.1) then implies that

$$
\lim _{t \rightarrow+\infty} \frac{h(t)}{t}=\lim _{t \rightarrow+\infty} h^{\prime}(t)=0
$$

and consequently, there exists a $t_{2}, t_{2} \geqq t_{1}$, dependent on $\hat{\beta}$ and hence on $\delta$, such that for all $t \geqq t_{2}$, the segment $m(t)$ is a segment of the nonnegative real axis, i.e.,

$$
m(t) \subset[0,+\infty), \text { for all } t \geqq t_{2} .
$$

Next, for any $\mu \geqq 1$ and for any $t \geqq t_{2}$, let $m_{\mu}(t)$ denote the following ellipse in the complex plane:

$$
m_{\mu}(t):=\left\{z=x+i y: \frac{(x-t)^{2}}{a^{2}}+\frac{y^{2}}{b^{2}}=1\right\},
$$

where

$$
\left\{\begin{array}{l}
a=a(t, \hat{\beta}, \mu):=\frac{\hat{\beta} h(t)}{2}\left(\mu+\mu^{-1}\right), \\
b=b(t, \hat{\beta}, \mu):=\frac{\hat{\beta} h(t)}{2}\left(\mu-\mu^{-1}\right),
\end{array}\right.
$$

and let $\bar{m}_{\mu}(t)$ denote the closed interior of $m_{\mu}(t)$, i.e.,

$$
\bar{m}_{\mu}(t):=\left\{z=x+i y: \frac{(x-t)^{2}}{a^{2}}+\frac{y^{2}}{b^{2}} \leqq 1\right\} .
$$

For each $t \geqq t_{2}$, let $A(t)$ denote the largest value of $\mu \geqq 1$ such that

$$
\bar{m}_{\mu}(t) \subset E_{1}(h)
$$


We claim that

$$
\lim _{t \rightarrow+\infty} A(t)=A^{*}(\hat{\beta})
$$

To prove (4.11) we establish upper and lower bounds for $A(t)$. Geometrically, the value for $\mu$ derived by setting $b=h(t)$ is obviously an upper bound for $A(t)$. But, using (4.9), this implies that

$$
\frac{\hat{\beta} h(t)}{2}\left(\mu-\mu^{-1}\right)=h(t)
$$

and, upon solving for $\mu$, it follows (cf. (4.4)) that

$$
\mu=A^{*}(\hat{\beta})
$$

Thus

$$
A(t) \leqq A^{*}(\hat{\beta}), \text { for each } t \geqq t_{2} \text {. }
$$

Next, since $h \in \mathscr{H}$, the function $h(t)$ is nondecreasing for, say, all $t \geqq t_{3} \geqq t_{2}$. Thus, the value for $\mu$ derived by setting $b=h(t-a)$ is again seen geometrically to be a lower bound for $A(t)$, i.e., from (4.9)

$$
\frac{\hat{\beta} h(t)}{2}\left(\mu-\frac{1}{\mu}\right)=h\left\{t-\frac{\hat{\beta} h(t)}{2}\left(\mu+\frac{1}{\mu}\right)\right\} .
$$

It is not difficult to see in fact that (4.12) has a unique solution $\mu^{*}(t)$ satisfying $1<\mu^{*}(t) \leqq A^{*}(\hat{\beta})$ for all $t$ sufficiently large. Indeed, on the interval $1 \leqq \mu \leqq A^{*}(\hat{\beta})$ it is clear from (4.8) that the right-hand side of (4.12) is a well-defined positive nonincreasing function of $\mu$ for all $t$, say, with $t \geqq t_{4} \geqq t_{3}$, while the left-hand side of (4.12) is a strictly increasing function of $\mu$. Moreover, for $\mu=1$ the left-hand side vanishes and is thus less than the positive value of the right-hand side for $\mu=1$, while for $\mu=A^{*}(\hat{\beta})$, the left-hand side reduces by definition to $h(t)$, which cannot be less than the corresponding value of the right-hand side for $\mu=$ $A^{*}(\hat{\beta})$ since $h(t)$ is nondecreasing for $t \geqq t_{3}$. Thus, there exists a unique solution $\mu^{*}(t)$ of $(4.12)$ which satisfies $1<\mu^{*}(t) \leqq A^{*}(\hat{\beta})$, and $\mu^{*}(t)$ is a lower bound for $A(t)$, i.e.,

$$
\mu^{*}(t) \leqq A(t) \leqq A^{*}(\hat{\beta}), \text { for all } t \geqq t_{4}
$$

We now show that $\mu^{*}(t)$, the unique solution of (4.12), satisfies $\lim _{t \rightarrow+\infty} \mu^{*}(t)=A^{*}(\hat{\beta})$. As $\mu^{*}(t)$ is bounded, i.e., $\mu^{*}(t) \leqq A^{*}(\hat{\beta})$, we see from (4.8) that 


$$
t-\frac{\hat{\beta} h(t)}{2}\left(\mu^{*}(t)+\frac{1}{\mu^{*}(t)}\right) \rightarrow+\infty \quad \text { as } \quad t \rightarrow+\infty .
$$

Therefore, since $h^{\prime}$ exists for all sufficiently large values of the argument, we can apply the Mean Value Theorem to deduce

$$
\begin{aligned}
h(t) & -h\left\{t-\frac{\hat{\beta} h(t)}{2}\left(\mu^{*}(t)+\frac{1}{\mu^{*}(t)}\right)\right\} \\
& =h^{\prime}\left(c_{t}\right) \frac{\hat{\beta} h(t)}{2}\left(\mu^{*}(t)+\frac{1}{\mu^{*}(t)}\right),
\end{aligned}
$$

where

$$
t-\frac{\hat{\beta} h(t)}{2}\left(\mu^{*}(t)+\left(\mu^{*}(t)\right)^{-1}\right)<c_{t}<t, \text { for all } t \text { large. }
$$

Dividing the expression in (4.13) by $h(t)$ and observing that $\lim _{t \rightarrow+\infty} c_{t}=+\infty$, it follows from hypothesis (2.1) that

$$
1-\frac{h\left\{t-\frac{\hat{\beta} h(t)}{2}\left(\mu^{*}(t)+\left(\mu^{*}(t)\right)^{-1}\right)\right\}}{h(t)}=h^{\prime}\left(c_{t}\right) \frac{\hat{\beta}}{2}\left(\mu^{*}(t)+\left(\mu^{*}(t)\right)^{-1}\right) \rightarrow 0
$$

as $t \rightarrow+\infty$. But, using the defining relation (4.12) for $\mu^{*}(t)$, the above gives us that

$$
\lim _{t \rightarrow+\infty} \frac{\hat{\beta}}{2}\left\{\mu^{*}(t)-\left(\mu^{*}(t)\right)^{-1}\right\}=1
$$

which implies, from the definition of $A^{*}(\beta)$ in (4.4), that

$$
\lim _{t \rightarrow+\infty} \mu^{*}(t)=A^{*}(\hat{\beta})
$$

Hence, as $\mu^{*}(t) \leqq A(t) \leqq A^{*}(\hat{\beta})$ for all $t \geqq t_{4}$, then evidently $\lim _{t \rightarrow+\infty} A(t)=A^{*}(\hat{\beta})$, as claimed in (4.11).

In order to "fill out" the region $E_{d}(h)$, where $d$ is fixed and satisfies (2.4), it suffices to work with ellipses $m_{\mu}(t)$, defined by setting

$$
b=d h(t) \text {. }
$$

From (4.9) and (4.4), the corresponding value of $\mu$ turns out to be $\mu=\rho^{*}(\hat{\beta}) \equiv A^{*}(\hat{\beta} / d)$. Geometrically, this implies that the elliptical 
region $\bar{m}_{\rho^{*}(\hat{\beta})}(t)$ covers the vertical segment $\{z=x+i y: x=t$ and $|y| \leqq$ $d h(t)\}$ of $E_{d}(h)$. Next, using (4.11) and the fact that $0<d<1$, then $1 \leqq \rho^{*}(\hat{\beta}) \equiv A^{*}(\hat{\beta} / d)<A(t)$ for all $t$ sufficiently large, say $t \geqq t_{5} \geqq t_{2}$. But from (4.7), we trivially have, since $m(t) \subset[0,+\infty)$ for all $t \geqq t_{2}$, that

$$
\left\|r_{n}-r_{n-1}\right\|_{m(t)}<\frac{\left(q_{2}+1\right)}{q_{2}^{n}}, \text { for all } n>n_{0}, \quad \text { all } t \geqq t_{2}
$$

Next, since the interior of $E_{1}(h)$ by hypothesis contains no poles of $r_{n}$ for all $n$ sufficiently large, say $n \geqq n_{1} \geqq n_{0}$, the same is evidently true for $\left(r_{n}-r_{n-1}\right) \in \pi_{2 n-1,2 n-1}$. Thus, applying (4.2) of Walsh's Lemma 4.1 with $[-1,+1]$ replaced by $m(t)$, gives us that

$$
\left\|r_{n}(z)-r_{n-1}(z)\right\|_{\hat{m}_{p}^{*}(\hat{\beta})(t)} \leqq \frac{\left(q_{2}+1\right)}{q_{2}^{n}}\left\{\frac{A(t) \rho^{*}(\hat{\beta})-1}{A(t)-\rho^{*}(\hat{\beta})}\right\}^{2 n-1}
$$

for all $n>n_{1}$, all $t \geqq t_{5}$. Since $\lim _{t \rightarrow \infty} A(t)=A^{*}(\hat{\beta})$ from (4.11), it follows from (4.6) that, for all $n>n_{1}$ and all $t$ sufficiently large, say $t \geqq t_{6} \geqq t_{5}$,

$$
\left\|r_{n}(z)-r_{n-1}(z)\right\|_{\left.\bar{m}_{p} \cdot \hat{\beta}\right)(t)} \leqq \frac{\left(q_{2}+1\right)}{q_{1}^{n}}\left(\frac{1+d}{1-d}\right)^{2 n}
$$

where $t_{6}$ is dependent on $\delta$. But, since $\bar{m}_{\rho^{*}(\hat{\beta})}(t)$ covers the vertical segment of $E_{d}(h)$ with abscissa $t$, then the set $\bigcup_{t \geqq t_{6}} \bar{m}_{\rho^{*}(\hat{\beta})}(t)$ evidently covers $E_{d}(h)$, with the possible exception of a bounded subset $K_{d}$ of $E_{d}(h)$, where $K_{d}$ depends upon the choice of $\delta$, as well as $d$. Hence,

$$
\left\|r_{n}(z)-r_{n-1}(z)\right\|_{E_{d}(h)-K_{d}} \leqq \frac{\left(q_{2}+1\right)}{q_{1}^{n}}\left(\frac{1+d}{1-d}\right)^{2 n} \text { for all } n>n_{1}
$$

which implies that the sequence $\left\{r_{n}(z)\right\}_{n=1}^{\infty}$ converges geometrically on $E_{d}(h)-K_{d}$ to an analytic extension $F(z)$ of $f(x)$, defined by means of

$$
F(z):=r_{n_{1}}(z)+\sum_{n=n_{1}}^{\infty}\left(r_{n+1}(z)-r_{n}(z)\right)
$$

Indeed, it follows from (4.15) that $\left\|r_{n-1+s}(z)-r_{n-1}(z)\right\|_{E_{d}(h)-K_{d}} \leqq \frac{\left(q_{2}+1\right)}{(1-\gamma) q_{1}^{n}}\left(\frac{1+d}{1-d}\right)^{2 n}$, for all $n>n_{1}$, all $s \geqq 1$, 


$$
\gamma:=\frac{1}{q_{1}}\left(\frac{1+d}{1-d}\right)^{2}<\frac{1}{q}\left(\frac{1+d}{1-d}\right)^{2}<1,
$$

so that letting $s \rightarrow \infty$,

$$
\left\|F(z)-r_{n-1}(z)\right\|_{E_{d}(h)-K_{d}} \leqq \frac{\left(q_{2}+1\right)}{(1-\gamma) q_{1}^{n}}\left(\frac{1+d}{1-d}\right)^{2 n}, \text { for all } n>n_{1} .
$$

This in turn implies that

$$
\varlimsup_{n \rightarrow \infty}\left\{\left\|F(z)-r_{n}(z)\right\|_{E_{d}(h)-K_{d}}\right\}^{1 / n}<\frac{1}{q}\left(\frac{1+d}{1-d}\right)^{2},
$$

which is the desired final inequality, (2.5), of Theorem 2.1.

Proof of Corollary 2.2. The existence of the entire function $G(z)$, of finite order, with $G(x)=g(x)$ for all $x \geqq 0$ is a consequence of [7, Theorem 3], even though the result given there is only stated for the case when $g$ is real on $[0,+\infty)$. Moreover, a careful examination of the proof of Theorem 3 of [7] shows that the sequence $\left\{p_{n}(z)\right\}_{n=1}^{\infty}$ converges uniformly and geometrically to $G(z)$ on any bounded set $T$ in the complex plane, the degree of convergence on $T$ being at least $1 / q$, i.e.,

$$
\varlimsup_{n \rightarrow \infty}\left\{\left\|G(z)-p_{n}(z)\right\|_{T}\right\}^{1 / n} \leqq \frac{1}{q} .
$$

Next, as no $p_{n}$ by hypothesis has zeros in the interior of $E_{1}(h)$ for all $n$ sufficiently large, the uniform convergence of $\left\{p_{n}\right\}_{n=1}^{\infty}$ to $G$ on any bounded set gives us that $G$ is nonzero at any interior point of $E_{1}(h)$ by Hurwitz's Theorem. Also, because $h(x)>0$ for all $x>0$, it is clear from the definition of the set $E_{\mathrm{s}}(h)$ in (2.2) that every point in $E_{d}(h)$, for each $d$ with $0<d<1$, is an interior point of $E_{1}(h)$, except for the vertical segment $\{z=i y:|y| \leqq d h(0)\}$. Hence, since $G(z)$ is given to be nonzero on this segment, then $G(z)$ is nonzero on all of $E_{d}(h)$. We remark that if $h(0)=0$, then the assumption that $G$ is nonzero on the segment $\{z=i y:|y| \leqq d h(0)\}$ reduces simply to the assumption that $G(0)=$ $g(0) \neq 0$.

We are now in a position to apply Theorem 2.1 with $f(x) \equiv 1 / g(x)$ for all $x \geqq 0$, and with $r_{n}=1 / p_{n}$ for all $n \geqq 1$. First, fix any $d$ which satisfies (2.4), and let $q^{\prime}$ be any number with $1<q^{\prime}<q$ such that $d<\left(\sqrt{q^{\prime}}-1\right) /\left(\sqrt{q^{\prime}}+1\right)$. Then, it follows from (2.6) that

$$
\varlimsup_{n \rightarrow \infty}\left\{\left\|\frac{1}{g(x)}-\frac{1}{p_{n}(x)}\right\|_{[0,+\infty)}\right\}^{1 / n}<\frac{1}{q^{\prime}}<1 .
$$


Hence, from (2.5) of Theorem 2.1, there exists a bounded subset $K_{d}$ of $E_{d}(h)$ such that

$$
\varlimsup_{n \rightarrow \infty}\left\{\left\|\frac{1}{G(z)}-\frac{1}{p_{n}(z)}\right\|_{E_{d}(h)-K_{d}}\right\}^{1 / n}<\frac{1}{q^{\prime}}\left(\frac{1+d}{1-d}\right)^{2}<1 .
$$

Now, since $G(z)$ is nonzero on $E_{d}(h)$, it is evidently nonzero on the closure of its bounded subset $K_{d}$. Thus, it follows from (4.16) with $T=K_{d}$ that

$$
\varlimsup_{n \rightarrow \infty}\left\{\left\|\frac{1}{G(z)}-\frac{1}{p_{n}(z)}\right\|_{K_{d}}\right\}^{1 / n} \leqq \frac{1}{q}<\frac{1}{q^{\prime}}<1 .
$$

Then, simply combining the results of (4.17) and (4.18) gives us that

$$
\varlimsup_{n \rightarrow \infty}\left\{\left\|\frac{1}{G(z)}-\frac{1}{p_{n}(z)}\right\|_{E_{d}(h)}\right\}^{1 / n} \leqq \frac{1}{q^{\prime}}\left(\frac{1+d}{1-d}\right)^{2}<1 .
$$

But as this inequality holds for all $q^{\prime}$ sufficiently close to $q$ with $1<q^{\prime}<q$, then

$$
\varlimsup_{n \rightarrow \infty}\left\{\left\|\frac{1}{G(z)}-\frac{1}{p_{n}(z)}\right\|_{E_{d}(h)}\right\}^{1 / n} \leqq \frac{1}{q}\left(\frac{1+d}{1-d}\right)^{2}<1,
$$

the desired result of (2.7).

Proof of Corollary 2.3. Because $\mathscr{C}$ is, from (2.13), contained in every $E_{d}(h), 0<d$, except for some compact set, it follows from (4.16) and (2.7) that

$$
\varlimsup_{n \rightarrow \infty}\left\{\left\|\frac{1}{G(z)}-\frac{1}{p_{n}(z)}\right\|_{6}\right\}^{1 / n} \leqq \frac{1}{q}\left(\frac{1+d}{1-d}\right)^{2}<1,
$$

for each $d>0$ sufficiently small. But as $\mathscr{C}$ is independent of $d$, then letting $d \rightarrow 0$ in the above inequality yields

$$
\varlimsup_{n \rightarrow \infty}\left\{\left\|\frac{1}{G(z)}-\frac{1}{p_{n}(z)}\right\|_{\mathscr{\ell}}\right\}^{1 / n} \leqq \frac{1}{q}<1
$$

On the other hand, the ray $[0,+\infty)$ is a subset of $\mathscr{C}$, so that

$$
\sigma:=\varlimsup_{n \rightarrow \infty}\left\{\left\|\frac{1}{g(x)}-\frac{1}{p_{n}(x)}\right\|_{(0,+\infty)}\right\}^{1 / n} \leqq \varlimsup_{n \rightarrow \infty}\left\{\left\|\frac{1}{G(z)}-\frac{1}{p_{n}(z)}\right\|_{\zeta}\right\}^{1 / n} \leqq \frac{1}{q} .
$$


Thus, if $0<\sigma<1$, we can choose $q$ from (2.6) with $1<q<+\infty$ so that $q=1 / \sigma$, and equality holds throughout in the above expression, which yields the desired result of (2.15). If, however $\sigma=0$, then (2.6) is valid for every $q>1$, and letting $q \rightarrow+\infty$ in the above expression again gives (2.15).

For the proofs of the theorems on overconvergence in regions having a positive angle at infinity, it is convenient to first establish

LEMMA 4.2. Let $\pi / 2 \leqq \phi_{1}<\phi_{2} \leqq \pi$, and, for $i=1,2$, let $\Delta^{*}\left(\phi_{1}, \rho\right)$ denote generically the open circular sector (in the $w$-plane) with vertex -1 , radius $\rho$, and opening $2 \phi_{i}(\geqq \pi)$ symmetric about the ray $[-1,+\infty)$, i.e.,

$$
\Delta^{*}\left(\phi_{l}, \rho\right):=\left\{w:|\arg (w+1)|<\phi_{\imath},|w+1|<\rho\right\} ;
$$

its closure being denoted by $\bar{\Delta}^{*}\left(\phi_{1}, \rho\right)$. Then, for each pair of positive numbers $\epsilon, \eta$, there exists a $\delta>0$ such that the inequality

$$
\left|\frac{1-\bar{\alpha} w}{w-\alpha}\right|<\frac{\sin \frac{1}{2}\left(\phi_{2}+\phi_{1}-\pi\right)}{\sin \frac{1}{2}\left(\phi_{2}-\phi_{1}\right)}+\epsilon
$$

holds whenever $w \in \bar{\Delta}^{*}\left(\phi_{1}, \delta\right)$ and $\alpha \notin \Delta^{*}\left(\phi_{2}, \eta\right), \alpha \neq-1$.

Proof. First, we fix $\alpha$ in the infinite sector $P:=\left\{w: \phi_{2} \leqq\right.$ $\left.\arg (w+1) \leqq 2 \pi-\phi_{2}\right\}$ which is contained in the complement of $\Delta^{*}\left(\phi_{2}, \eta\right)$, i.e.,

$$
\alpha=-1+r e^{i \theta}, \quad r>0, \quad \phi_{2} \leqq \theta \leqq 2 \pi-\phi_{2},
$$

and we consider the image of the closed infinite sector $S:=$ $\left\{w:|\arg (w+1)| \leqq \phi_{1}\right\}$ under the bilinear transformation

$$
\xi=T(w):=\frac{1-\bar{\alpha} w}{w-\alpha}
$$

Let $\mathscr{L}^{+}$and $\mathscr{L}^{-}$denote, respectively, the lines

$$
\begin{aligned}
& \mathscr{L}^{+}:=\left\{w: \arg (w+1)=\phi_{1} \text { or } \phi_{1}+\pi\right\} ; \\
& \mathscr{L}^{-}:=\left\{w: \arg (w+1)=\pi-\phi_{1} \text { or } 2 \pi-\phi_{1}\right\} .
\end{aligned}
$$

Since these lines do not pass through $\alpha$, they are mapped by $T$ to true (intersecting) circles $C_{\alpha}^{+}$and $C_{\alpha}^{-}$in the $\xi$-plane. Furthermore, since $\alpha \notin S$, the image $T(S)$ is the union of the closed interiors of these circles. 
To estimate the moduli of points on $C_{\alpha}^{+}$, we find the center $c_{\alpha}^{+}$and radius $r_{\alpha}^{+}$of this circle. As $T(\alpha)=\infty$, we have $c_{\alpha}^{+}=T\left(\alpha^{*}\right)$, where

$$
\alpha^{*}=-1+r e^{l\left(2 \phi_{1}-\theta\right)}
$$

is the reflection of $\alpha$ in the line $\mathscr{L}^{+}$. Thus,

$$
c_{a}^{+}=\frac{1-\bar{\alpha} \alpha^{*}}{\alpha^{*}-\alpha}=\frac{e^{i\left(2 \phi_{1}-\theta\right)}+e^{-i \theta}-r e^{2 \ell\left(\phi_{1}-\theta\right)}}{e^{i\left(2 \phi_{1}-\theta\right)}-e^{i \theta}},
$$

and so,

$$
\left|c_{\alpha}^{+}\right| \leqq \frac{-\cos \phi_{1}}{\sin \left(\theta-\phi_{1}\right)}+\frac{r}{2 \sin \left(\theta-\phi_{1}\right)} .
$$

Furthermore, since $T(\infty)=-\bar{\alpha} \in C_{t}^{+}$, we have

$$
r_{\alpha}^{+}=\left|c_{\alpha}^{+}+\bar{\alpha}\right|=\frac{|\alpha|^{2}-1}{\left|\alpha^{*}-\alpha\right|}=\frac{-\cos \theta}{\sin \left(\theta-\phi_{1}\right)}+\frac{r}{2 \sin \left(\theta-\phi_{1}\right)}
$$

Now, (4.20) and (4.21) imply that, for $\xi$ on $C_{\alpha}^{+}$,

$$
\begin{aligned}
|\xi| \leqq\left|c_{\alpha}^{+}\right|+r_{\alpha}^{+} & \leqq \frac{-\cos \phi_{1}-\cos \theta}{\sin \left(\theta-\phi_{1}\right)}+\frac{r}{\sin \left(\theta-\phi_{1}\right)} \\
& =\frac{\sin \frac{1}{2}\left(\theta+\phi_{1}-\pi\right)}{\sin \frac{1}{2}\left(\theta-\phi_{1}\right)}+\frac{r}{\sin \left(\theta-\phi_{1}\right)},
\end{aligned}
$$

and it is easy to verify that the next to last term is a nonincreasing function of $\theta$ on $\left[\phi_{2}, 2 \pi-\phi_{2}\right]$, and that $\sin \left(\theta-\phi_{1}\right) \geqq \sin \left(\phi_{2}-\phi_{1}\right)>0$. Hence,

$$
|\xi| \leqq \frac{\sin \frac{1}{2}\left(\phi_{2}+\phi_{1}-\pi\right)}{\sin \frac{1}{2}\left(\phi_{2}-\phi_{1}\right)}+\frac{r}{\sin \left(\phi_{2}-\phi_{1}\right)}, \quad \xi \in C_{\alpha}^{+}
$$

and, by a symmetry argument, the same inequality holds for $\xi \in C_{\alpha}^{-}$. Therefore, for any $w \in S, \alpha \in P$, and $|\alpha+1| \leqq r$, we have

$$
\left|\frac{1-\bar{\alpha} w}{w-\alpha}\right| \leqq \frac{\sin \frac{1}{2}\left(\phi_{2}+\phi_{1}-\pi\right)}{\sin \frac{1}{2}\left(\phi_{2}-\phi_{1}\right)}+\frac{r}{\sin \left(\phi_{2}-\phi_{1}\right)} .
$$

Consequently, given $\epsilon$ and $\eta$, fix $\tau=\min \left\{\eta, \epsilon \cdot \sin \left(\phi_{2}-\phi_{1}\right)\right\}$, so that inequality (4.19) holds whenever $w \in S, \alpha \in P$, and $0<|\alpha+1|<\tau$.

Finally, assume $|\alpha+1| \geqq \tau$ and suppose that $w \in S$ with $|w+1| \leqq$ $\delta<\tau$. It is easy to verify that 


$$
\begin{aligned}
\left|\frac{1-\bar{\alpha} w}{w-\alpha}\right| & =\left|w-\frac{w+1}{\bar{\alpha}+1}\right| \cdot\left|1-\frac{w+1}{(w+1)-(\alpha+1)}\right| \\
& \leqq\left\{|w|+\frac{\delta}{\tau}\right\} \cdot\left\{1+\frac{\delta}{\tau-\delta}\right\} .
\end{aligned}
$$

Writing $w=-1+\hat{\delta} e^{\imath \theta}, 0 \leqq \hat{\delta} \leqq \delta$, with $|\theta| \leqq \phi_{1}$, it follows that $|w|=$ $\left(1-2 \hat{\delta} \cos \theta+\hat{\delta}^{2}\right)^{1 / 2} \leqq\left(1-2 \delta \cos \phi_{1}+\delta^{2}\right)^{1 / 2}$. Substituting this into the above display then yields

$$
\left|\frac{1-\bar{\alpha} w}{w-\alpha}\right| \leqq 1+\delta\left(\frac{2}{\tau}-\cos \phi_{1}\right)+\mathscr{O}\left(\delta^{2}\right)<1+\epsilon
$$

for all $\delta$ sufficiently small. But, as the right side of (4.19) is greater than $1+\epsilon$, then (4.19) must hold for $w \in \bar{\Delta}^{*}\left(\phi_{1}, \delta\right)$ and $\alpha \notin \Delta^{*}\left(\phi_{2}, \eta\right), \alpha \neq-1$.

We now prove an overconvergence result for circular sectors with vertex at $z=-1$.

LEMMA 4.3. Assume that $f$, defined and finite on $[-1,1]$, is such that there exists a sequence of rational functions $\left\{r_{n}\right\}_{n=1}^{\infty}$, with $r_{n} \in \pi_{n, n}$ for all $n \geqq 1$, and a real number $q>1$ such that

$$
\varlimsup_{n \rightarrow \infty}\left\{\left\|f-r_{n}\right\|_{[-1,1]}\right\}^{1 / n}<\frac{1}{q}<1 .
$$

Let $\Delta(\phi, \rho)$ denote generically the open circular sector

$$
\Delta(\phi, \rho):=\{z:|\arg (z+1)|<\phi,|z+1|<\rho\}
$$

and assume that $\Delta\left(\theta_{0}, \mu_{0}\right)$, where $0<\theta_{0} \leqq \pi$ and $\mu_{0}>0$, contains no poles of the $r_{n}(z)$ for all $n$ sufficiently large. Then, for each fixed $\theta, 0<\theta<\theta_{0}$, satisfying

$$
\frac{\sin \frac{1}{4}\left(\theta_{0}+\theta\right)}{\sin \frac{1}{4}\left(\theta_{0}-\theta\right)}<\sqrt{q},
$$

i.e.

$$
0<\theta<4 \tan ^{-1}\left\{\left(\frac{\sqrt{q}-1}{\sqrt{q}+1}\right) \cdot \tan \left(\frac{\theta_{0}}{4}\right)\right\}
$$

there exists a $\mu=\mu(\theta)>0$ and a function $F(z)$ analytic on $\Delta(\theta, \mu)$, continuous on the closure $\bar{\Delta}(\theta, \mu)$, with $F(x)=f(x)$ for all $x$ of $[-1,+1]$ 
in $[-1,-1+\mu]$, such that $\left\{r_{n}(z)\right\}_{n=1}^{\infty}$ converges geometrically to $F$ on $\bar{\Delta}(\theta, \mu)$. Moreover,

$$
\varlimsup_{n \rightarrow \infty}\left\{\left\|F-r_{n}\right\|_{\bar{\Delta}(\theta, \mu)}\right\}^{1 / n}<\frac{1}{q}\left\{\frac{\sin \frac{1}{4}\left(\theta_{0}+\theta\right)}{\sin \frac{1}{4}\left(\theta_{0}-\theta\right)}\right\}^{2}<1 .
$$

Proof. First, choose $q_{1}>q$ so that

$$
\varlimsup_{n \rightarrow \infty}\left\{\left\|f-r_{n}\right\|_{[-1,1]}\right\}^{1 / n}<\frac{1}{q_{1}}<\frac{1}{q},
$$

and let $\gamma$ satisfy

$$
1<\gamma<\left(q_{1} / q\right)^{1 / 4}
$$

From (4.26) and the triangle inequality, we have for $n$ sufficiently large, say $n \geqq n_{0}$, that

$$
\left\|r_{n}-r_{n-1}\right\|_{[-1,1]}<\frac{\left(q_{1}+1\right)}{q_{1}^{n}}
$$

Let $\theta$ be fixed and satisfy (4.23), and choose $\hat{\theta}_{0}$ and $\hat{\theta}$ so that

$$
\theta<\hat{\theta}<\hat{\theta}_{0}<\theta_{0}, \quad \text { and } \quad \frac{\sin \frac{1}{4}\left(\hat{\theta}_{0}+\hat{\theta}\right)}{\sin \frac{1}{4}\left(\hat{\theta}_{0}-\hat{\theta}\right)}<\gamma \cdot \frac{\sin \frac{1}{4}\left(\theta_{0}+\theta\right)}{\sin \frac{1}{4}\left(\theta_{0}-\theta\right)}
$$

Since the derivative of the function $\Psi(w)$ of Walsh's Lemma 4.1 has a simple zero at $w=-1$, an angle $\theta$ at $z=-1$ is sent into $\frac{1}{2}(\theta+\pi)$ by the inverse mapping $w=\Phi(z)$. Consequently, as $\hat{\theta}_{0}<\theta_{0}$, the image of the set $\Delta\left(\theta_{0}, \mu_{0}\right)-[-1,1]$ under $\Phi$ must, for some $\eta>0$, contain the set

$$
\Delta^{*}\left(\frac{\hat{\theta}_{0}}{2}+\frac{\pi}{2}, \eta\right) \cap\{w:|w|>1\}
$$

where the asterisk denotes that the circular sector is in the $w$ plane. Since $\gamma>1$, Lemma 4.2 (with $\phi_{1}=\hat{\theta} / 2+\pi / 2, \phi_{2}=\hat{\theta}_{0} / 2+\pi / 2$ ) implies that there exists a $\delta>0$ such that the inequality

$$
\left|\frac{1-\overline{\Phi(\beta)} \cdot \Phi(z)}{\Phi(z)-\Phi(\beta)}\right|<\gamma \cdot \frac{\sin \frac{1}{4}\left(\hat{\theta}_{0}+\hat{\theta}\right)}{\sin \frac{1}{4}\left(\hat{\theta}_{0}-\hat{\theta}\right)}
$$

holds whenever $\beta \notin \Delta\left(\theta_{0}, \mu_{0}\right), \beta \notin[-1,1]$, and 


$$
w=\Phi(z) \in \bar{\Delta}^{*}\left(\frac{\hat{\theta}}{2}+\frac{\pi}{2}, \delta\right)
$$

But, as $\hat{\theta}>\theta$, we can find a $\mu, 0<\mu<2$, so that (4.31) holds for all $z \in \bar{\Delta}(\theta, \mu)-[-1,1]$. Now, by hypothesis, no poles of $r_{n}(z)-r_{n-1}(z)$ lie in $\Delta\left(\theta_{0}, \mu_{0}\right)$ for all $n$ large, say $n \geqq n_{1} \geqq n_{0}$, and thus, from (4.28), (4.30), and (4.1) of Lemma 4.1, we deduce that

$$
\left\|r_{n}-r_{n-1}\right\|_{\bar{\Delta}(\theta, \mu)} \leqq \frac{\left(q_{1}+1\right)}{q_{1}^{n}}\left\{\gamma \cdot \frac{\sin \frac{1}{4}\left(\hat{\theta}_{0}+\hat{\theta}\right)}{\sin \frac{1}{4}\left(\hat{\theta}_{0}-\hat{\theta}\right)}\right\}^{2 n-1},
$$

for $n>n_{1}$. Hence, by the argument used in the proof of Theorem 2.1, the sequence $\left\{r_{n}(z)\right\}_{n=1}^{\infty}$ converges geometrically on $\bar{\Delta}(\theta, \mu)$ to an analytic extension $F(z)$ of $f(x)$. Furthermore, from (4.32), (4.29), and (4.27), we have

$$
\begin{aligned}
\varlimsup_{n \rightarrow \infty}\left\{\left\|F-r_{n}\right\|_{\bar{\Delta}(\theta, \mu)}\right\}^{1 / n} & \leqq \frac{1}{q_{1}}\left\{\gamma \cdot \frac{\sin \frac{1}{4}\left(\hat{\theta}_{0}+\hat{\theta}\right)}{\sin \frac{1}{4}\left(\hat{\theta}_{0}-\hat{\theta}\right)}\right\}^{2} \\
& <\frac{1}{q}\left\{\frac{\sin \frac{1}{4}\left(\theta_{0}+\theta\right)}{\sin \frac{1}{4}\left(\theta_{0}-\theta\right)}\right\}^{2}<1
\end{aligned}
$$

which is the desired inequality (4.25).

Proof of Theorem 2.4. Observe that Lemma 4.3 trivially remains valid if the line segments of the circular sectors $\Delta\left(\theta_{0}, \mu_{0}\right)$ and $\Delta(\theta, \mu)$ are replaced by smooth arcs making angles of $\theta_{0}$ and $\theta$, respectively, with the segment $[-1,1]$ at $z=-1$. Also, condition (4.22) is invariant under bilinear transformations of $[-1,1]$, and therefore so is Lemma 4.3. As a consequence of these facts we obtain Theorem 2.4.

The proofs of Corollaries 2.5 and 2.6 are similar to those of Corollaries 2.2 and 2.3, and hence we omit them.

Proof of Theorem 2.7. Applying Lemma 4.3 (with the interval $[-1,1]$ translated to the interval $[0,2])$, and applying Theorem 2.4 , we know that there exists a $\theta^{*}>0$ and a $\rho, 1<\rho<\infty$, such that the sequence $\left\{r_{n}(z)\right\}_{n=1}^{\infty}$ converges geometrically on the set

$$
A:=\bar{S}\left(\theta^{*}\right)-\{1 / \rho \leqq|z| \leqq \rho\} .
$$

But, as the $r_{n}(z)$ (trivially) converge geometrically on the segment $[1 / \rho, \rho]$, and have poles a positive distance (independent of $n$ ) from this segment, it follows from a theorem of Walsh (see $\S 9.8$ of [18]) that the 
$r_{n}(z)$ must converge geometrically in the closed interior of some nondegenerate ellipse $\mathscr{E}$ with foci at $1 / \rho$ and $\rho$. Consequently, by choosing $\theta, \theta^{*}>\theta>0$, so small that $\bar{S}(\theta) \subset A \cup \mathscr{E}$, the sequence of rational functions will converge geometrically on $\bar{S}(\theta)$, necessarily to an analytic extension $F$ of $f$.

Concerning the proof of Corollary 2.8 we remark that if rational functions $r_{n}(z)$ converge faster than geometrically on $[0,+\infty)$, then it is known (see Aharonov and Walsh [1], and Gončar [5]) that the $r_{n}(z)$ converge uniformly on any closed bounded set $K$ of the plane which contains no limit points of the poles of the $r_{n}(z)$, the convergence on $K$ being faster than geometrically.

Proof of Corollary 2.8. In this case, inequality (2.18) holds for every $q>1$. Hence, given $\theta, 0<\theta<\theta_{0}$, and given $\epsilon>0$, we can choose $q$ so large that

$$
\frac{1}{q}\left\{\frac{\sin \frac{1}{4}\left(\theta_{0}+\theta\right)}{\sin \frac{1}{4}\left(\theta_{0}-\theta\right)}\right\}^{2}<\epsilon
$$

and so (as in the proof of Theorem 2.7) there exists a $\rho=\rho(\epsilon)>1$ such that

$$
\varlimsup_{n \rightarrow \infty}\left\{\left\|F-r_{n}\right\|_{\bar{s}(\theta)-K}\right\}^{1 / n}<\epsilon
$$

where

$$
K:=\{z \in \bar{S}(\theta): 1 / \rho \leqq|z| \leqq \rho\}
$$

Since, for all $n$ large, $K$ is a positive distance from the poles of the $r_{n}(z)$, we have by the result of [1] and [5] mentioned above that

$$
\lim _{n \rightarrow \infty}\left\{\left\|F-r_{n}\right\|_{K}\right\}^{1 / n}=0
$$

Therefore, from (4.33) and (4.34) we obtain

$$
\varlimsup_{n \rightarrow \infty}\left\{\left\|F-r_{n}\right\|_{\bar{s}(\theta)}\right\}^{1 / n}<\epsilon,
$$

and as $\epsilon>0$ is arbitrary, equation (2.24) follows.

This completes the proof of the main results stated in $\$ 2$. It remains to verify Proposition 3.5. The proof requires three lemmas, the first of which concerns approximation on the ray $[0,+\infty)$. 
Lemma 4.4. Under the assumptions of Proposition 3.5

$$
\lim _{n \rightarrow \infty}\left\{\left\|\frac{1}{g(x)}-\frac{1}{s_{n}(x)}\right\|_{[0,+\infty)}\right\}^{1 / n^{2}}=\frac{1}{\sqrt{a}} .
$$

The proof of (4.35) follows from a straightforward modification of the argument used by Meinardus and Varga [8] in their proof of Theorem 3.1. See also Erdös and Reddy [4].

Lemma 4.5. Let T be any bounded set in the complex plane. Then, with the assumptions of Proposition 3.5,

$$
\varlimsup_{n \rightarrow \infty}\left\{\left\|g-s_{n}\right\|_{T}\right\}^{1 / n^{2}} \leqq \frac{1}{a} .
$$

Proof. If $\rho:=\sup \{|z|: z \in T\}$, then direct calculations show that

$$
\left|g(z)-s_{n}(z)\right| \leqq \sum_{k=n+1}^{\infty} \rho^{k} / a^{k^{2}} \leqq \frac{\rho^{n+1}}{a^{(n+1)^{2}}}\left(1-\frac{\rho}{a^{2(n+1)}}\right)^{-1}
$$

for all $n$ large and all $z \in T$, from which (4.36) directly follows.

The final lemma can be found in Pólya and Szegö ([9], vol. 2, p. 69, prob. 176).

Lemma 4.6. The function $g(z)$ and the partial sums $s_{n}(z)$ of Proposition 3.5 have all their zeros on the negative real axis.

Proof of Proposition 3.5. Using the above lemmas and applying the techniques of this section, it is easy to show that, for $1<q<\sqrt{a}$ and for $0<\theta<\pi$, the inequality

$$
\left\|\frac{1}{s_{n}}-\frac{1}{s_{n-1}}\right\|_{\bar{s}(\theta)} \leqq \frac{\left(q^{2 n+1}+1\right)}{q^{n^{2}}} \cdot \tau^{2 n-1}
$$

is valid for all $n$ sufficiently large, where $\tau>1$ is some constant independent of $n$.

This last inequality together with the arbitrariness of $q$ implies that

$$
\varlimsup_{n \rightarrow \infty}\left\{\left\|\frac{1}{g}-\frac{1}{s_{n}}\right\|_{\bar{s}(\theta)}\right\}^{1 / n^{2}} \leqq \frac{1}{\sqrt{a}} .
$$

But, by Lemma 4.4 , we also have 


$$
\frac{1}{\sqrt{a}}=\lim _{n \rightarrow \infty}\left\{\left\|\frac{1}{g}-\frac{1}{s_{n}}\right\|_{[0,+\infty)}\right\}^{1 / n^{2}} \leqq \frac{\lim }{n \rightarrow \infty}\left\{\left\|\frac{1}{g}-\frac{1}{s_{n}}\right\|_{\bar{s}(\theta)}\right\}^{1 / n^{2}},
$$

and so equation (3.13) follows.

\section{REFERENCES}

1. D. Aharonov and J. L. Walsh, On the convergence of rational functions of best approximation to a meromorphic function, J. Math. Anal. Appl., 40 (1972), 418-426.

2. Ralph P. Boas, Entire Functions, Academic Press, New York, 1954.

3. Fritz Carlson, Sur les fonctions entières, Arkiv for Mathematik, Astronomi O. Fysik, Bd. 35A, No. 14 (1948), 18 pp.

4. P. Erdös and A. R. Reddy, Rational Chebyshev approximation on the positive real axis, Notices of the Amer. Math. Soc., 21 (1974), p. A-159.

5. A. A. Gončar, On overconvergence of sequences of rational functions, Doklady Academii Nauk SSSR, 141 (1961), 1019-1022.

6. Günter Meinardus, Approximation on Functions: Theory and Numerical Methods, Springer Tracts in Natural Philosophy, Vol. 13, Springer-Verlag, New York, 1967.

7. G. Meinardus, A. R. Reddy, G. D. Taylor, and R. S. Varga, Converse theorems and extensions in Chebyshev rational approximation to certain entire functions in $[0,+\infty)$, Trans. Amer. Math. Soc., 170 (1972), 171-185.

8. Günter Meinardus and Richard S. Varga, Chebyshev rational approximations to certain entire functions in $[0,+\infty)$, J. Approximation Theory, 3 (1970), 300-309.

9. G. Pólya and G. Szegö, Aufgaben und Lehrsätze aus der Analysis, Springer-Verlag, Berlin, 1964.

10. J. A. Roulier and G. D. Taylor, Rational Chebyshev approximation on $[0,+\infty)$, J. Approximation Theory, 11 (1974), 208-215.

11. E. B. Saff and R. S. Varga, Convergence of Padé approximants to $e^{-z}$ on unbounded sets, J. Approximation Theory, 13 (1975), 470-488.

12. Z Zero-free parabolic regions for sequences of polynomials, SIAM J. Math. Anal., (to appear).

13. - On the zeros and poles of Padé approximants to $e^{z}$, Numer. Math., (to appear).

14. G. Szegö, Über eine Eigenschaft der Exponentialreihe, Berlin Math. Ges. Sitzungsber., 23 (1924), $50-64$.

15. Georges Valiron, Lectures on the General Theory of Integral Functions, Chelsea Publishing Co., New York, 1949.

16. Richard S. Varga, Semi-infinite and infinite strips free of zeros, Rendiconti del Seminario Matematico Università e Politechnico di Torino, 11 (1952), 289-296.

17. - Properties of a special set of entire functions and their respective partial sums, Ph.D. Thesis, Harvard University, 1954.

18. J. L. Walsh, Interpolation and Approximation by Rational Functions in the Complex Domain, Colloq. Publication Vol. 20, Amer. Math. Soc. Providence, R. I., fifth ed., 1969.

Received May 9, 1974 and in revised form October 21, 1975. The first author's research was supported in part by the Air Force Office of Scientific Research under Grant AFOSR-74-2688, and by the University of South Florida Research Council, and the second author's research was supported in part by the Air Force Office of Scientific Research under Grant AFOSR-74-2729, and by the Energy Research and Development Administration (ERDA) under Grant E(11-1)-2075.

UNIVERSITY OF SOUTH FLORIDA

AND

CALifornia Institute of TeChNology

Kent State University 



\section{PACIFIC JOURNAL OF MATHEMATICS}

\section{EDITORS}

RICHARD ARENS (Managing Editor)

University of California

Los Angeles, California 90024

\section{J. Dugundu}

Department of Mathematics University of Southern California Los Angeles, California 90007

D. Gilbarg and J. Milgram Stanford University Stanford, California 94305

\section{ASSOCIATE EDITORS}
E. F. BECKENBACH
B. H. NeumanN
F. WOLF
K. YoSHIDA

\section{SUPPORTING INSTITUTIONS}

UNIVERSITY OF BRITISH COLUMBIA CALIFORNIA INSTITUTE OF TECHNOLOGY

UNIVERSITY OF CALIFORNIA

MONTANA STATE UNIVERSITY

UNIVERSITY OF NEVADA

NEW MEXICO STATE UNIVERSITY

OREGON STATE UNIVERSITY

UNIVERSITY OF OREGON

OSAKA UNIVERSITY

\author{
UNIVERSITY OF SOUTHERN CALIFORNIA \\ STANFORD UNIVERSITY \\ UNIVERSITY OF HAWAII \\ UNIVERSITY OF TOKYO \\ UNIVERSITY OF UTAH \\ WASHINGTON STATE UNIVERSITY \\ UNIVERSITY OF WASHINGTON \\ AMERICAN MATHEMATICAL SOCIETY
}

The Supporting Institutions listed above contribute to the cost of publication of this Journal, but they are not owners or publishers and have no responsibility for its contents or policies.

Mathematical papers intended for publication in the Pacific Journal of Mathematics should be in typed form or offset-reproduced (not dittoed), double spaced with large margins. Underline Greek letters in red, German in green, and script in blue. The first paragraph or two must be capable of being used separately as a synopsis of the entire paper. Items of the bibliography should not be cited there unless absolutely necessary, in which case they must be identified by author and Journal, rather than by item number. Manuscripts, in duplicate, may be sent to any one of the four editors. Please classify according to the scheme of Math. Reviews, Index to Vol. 39. All other communications should be addressed to the managing editor, or Elaine Barth, University of California, Los Angeles, California, 90024.

100 reprints are provided free for each article, only if page charges have been substantially paid. Additional copies may be obtained at cost in multiples of 50 .

The Pacific Journal of Mathematics is issued monthly as of January 1966. Regular subscription rate: $\$ 72.00$ a year (6 Vols., 12 issues). Special rate: $\$ 36.00$ a year to individual members of supporting institutions.

Subscriptions, orders for back numbers, and changes of address should be sent to Pacific Journal of Mathematics, 103 Highland Boulevard, Berkeley, California, 94708.

PUBLISHED BY PACIFIC JOURNAL OF MATHEMATICS, A NON-PROFIT CORPORATION

Printed at Jerusalem Academic Press, POB 2390, Jerusalem, Israel.

$$
\begin{gathered}
\text { Copyright } 1976 \text { Pacific Journal of Mathematics } \\
\text { All Rights Reserved }
\end{gathered}
$$




\section{Pacific Journal of Mathematics}

Vol. 62, No. 2

February, 1976

Allan Russell Adler and Catarina Isabel Kiefe, Pseudofinite fields, procyclic

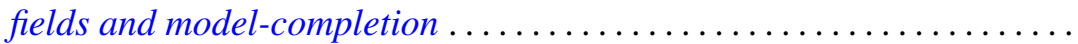

Christopher Allday, The stratification of compact connected Lie group

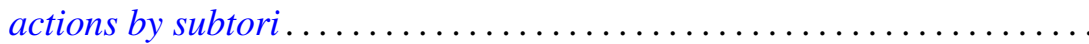

Martin Bartelt, Commutants of multipliers and translation operators .......

Herbert Stanley Bear, Jr., Ordered Gleason parts ..................

James Robert Boone, On irreducible spaces. II .....................

James Robert Boone, On the cardinality relationships between discrete

collections and open covers ............................

L. S. Dube, On finite Hankel transformation of generalized functions .......

Michael Freedman, Uniqueness theorems for taut submanifolds . . . . . . . . .

Shmuel Friedland and Raphael Loewy, Subspaces of symmetric matrices

containing matrices with a multiple first eigenvalue .............

Theodore William Gamelin, Uniform algebras spanned by Hartogs

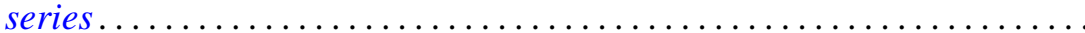

James Guyker, On partial isometries with no isometric part ............

Shigeru Hasegawa and Ryōtarō Satō, A general ratio ergodic theorem for

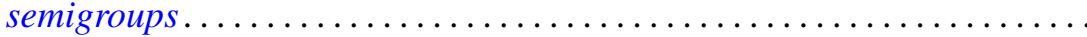

Nigel Kalton and G. V. Wood, Homomorphisms of group algebras with norm less than $\sqrt{2}$.

Thomas Laffey, On the structure of algebraic algebras...

Will Y. K. Lee, On a correctness class of the Bessel type differential operator $S_{\mu}$

Robert D. Little, Complex vector fields and divisible Chern classes ....

Kenneth Louden, Maximal quotient rings of ring extensions . .

Dieter Lutz, Scalar spectral operators, ordered $l^{\rho}$-direct sums, and the

counterexample of Kakutani-McCarthy . .

Ralph Tyrrell Rockafellar and Roger Jean-Baptiste Robert Wets, Stochastic

convex programming: singular multipliers and extended duality

singular multipliers and duality.

Edward Barry Saff and Richard Steven Varga, Geometric overconvergence of rational functions in unbounded domains ..........

Joel Linn Schiff, Isomorphisms between harmonic and P-harmonic Hardy

spaces on Riemann surfaces.

Virinda Mohan Sehgal and S. P. Singh, On a fixed point theorem of

Krasnoselskii for locally convex spaces.

Lewis Shilane, Filtered spaces admitting spectral sequence operations

Michel Smith, Generating large indecomposable continua . 